\title{
Acoustic detection of UHE neutrinos in the Mediterranean sea: status and perspective
}

\author{
Francesco Simeone ${ }^{1, \star}$ and Antonio Capone $e^{1,2}$ \\ ${ }^{1}$ INFN Roma1 \\ ${ }^{2}$ University "Sapienza", Roma
}

\begin{abstract}
In recent years the astro-particle community is involved in the realization of experimental apparatuses for the detection of high energy neutrinos originated in cosmic sources or produced in the interaction of Cosmic Rays with the Cosmic Microwave Background. For neutrino energies in the TeV-PeV range, optical Cherenkov detectors, that have been so far positively exploited by Baikal[1], IceCube[2] and ANTARES[3], are considered optimal. For higher energies, three different experimental techniques are under study: the detection of radio pulses produced by showers induced by a neutrino interaction, the detection of air showers initiated by neutrinos interacting with rocks or deep Earth's atmosphere and the detection of acoustic waves produced by deposition of energy following the interaction of neutrinos in an acoustically transparent medium. The potential of the acoustic detection technique, first proposed by Askaryan[4], to build very large neutrino detectors is appealing, thanks to the optimal properties of media such as water or ice as sound propagator. Though the studies on this technique are still in an early stage, acoustic positioning systems used to locate the optical modules in underwater Cherenkov neutrino detectors, give the possibility to study the ambient noise and provide important information for the future analysis of acoustic data.
\end{abstract}

\section{Introduction}

The interest in the study of neutrinos above $10^{17} \mathrm{eV}$, as a tool to investigate several open questions in the fields of high energy astro-particle physics and astrophysics, has grown steadily in recent years. Neutrinos created in cosmic sources or produced in the interaction of Ultra High Energy (UHE) protons with the cosmic microwave background via the Greisen-Zatsepin-Kuzmin mechanism[5], could provide complementary information to UHE charged cosmic ray and high energy gamma ray measurements. Moreover neutrinos are the only particle usable to probe the UHE non-thermal universe at distances above tens of megaparsec; all other particles are subject to interactions or decay, thus their use is limited to the local universe. Neutrino flux at these energies $\left(>10^{17} \mathrm{eV}\right)$, derived from cosmic ray[6] and gamma ray [7] measurements, is very small and the expected event rate is of the order of $0.1 \mathrm{~km}^{-2} \mathrm{yr}^{-1}$; detection of UHE neutrinos with a reasonable statistics may need a detector of at least $100 \mathrm{~km}^{3}$ size. The acoustic detection technique could be the basic ingredient to instrument such a huge volume with a reasonable number of sensors thanks to the large attenuation length $(\sim \mathrm{km})$ of the generated sound signal in water.

\footnotetext{
^e-mail: francesco.simeone@roma1.infn.it
} 


\section{The Thermo-Acoustic Model}

The acoustic detection technique of neutrino induced cascades in water is based on the thermoacoustic effect[4]; the cascade energy is deposited in a narrow region of the medium, it induces a local heating and results in a rapid expansion of the water. The expansion originates a pressure wave that propagates perpendicularly to the cascade direction, whose amplitude depends on the energy density deposited in the medium. Neutrinos interact with the nucleons of the medium through neutral or charge current interactions; in the NC interaction only the hadronic shower at the interaction point can be detected, in the CC interaction the lepton, created at the interaction point, releases its energy according to its nature; While for $v_{e}$ and in part for $v_{\tau}$, the lepton energy is deposited as a shower close to the interaction vertex, for $v_{\mu}$ the muon energy is deposited along its long track. In a wide range of energy useful for the acoustic detection, the ratio of the CC cross section[8] over the total is $\approx 70 \%$ (see [9]). As pointed out in [9] the mean inelasticity, so the fraction of the incident neutrino energy deposited in the induced hadronic shower and shown in Fig. (1), is weakly dependent on energy and its value is about $20 \%$. From these considerations it might seems that the golden events are the electromagnetic showers produced in CC interactions, which are the most abundant and retain about $80 \%$ of the incident neutrino energy. Unfortunately this is not the case at very high energy; it was first noted by Landau, Pomeranchuk and Migdal[10] that above a threshold of about $10^{17} \mathrm{eV}$, the cross sections for bremsstrahlung and pair production decrease as $\sqrt{E}$. As a consequence the cascade becomes longer and sub showers will develop along the main shower as the particle energy drops below the LMP threshold; this prevents the energy density to increase linearly with the neutrino energy: hence the charged lepton contribute little to the production of the acoustic signal. The longitudinal profile of one cascade above the LPM threshold[11] is shown in Fig. (1)
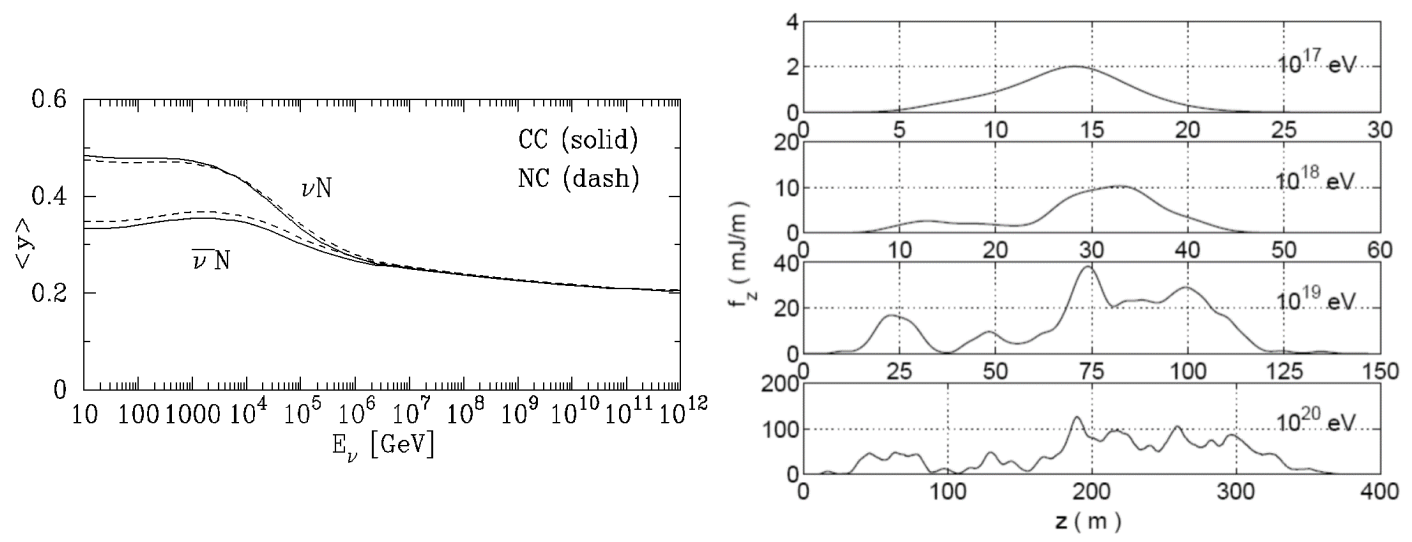

Figure 1. On left side the mean inelasticity as function of the neutrino energy and on the right the energy density profile of an electromagnetic cascade at different energy

Simulations of the acoustic signal generated by an hadronic shower in water has been performed by many authors, but uncertainty in the particle interaction and in the energy loss process at these energy as well as large fluctuation between showers, limit somehow the absolute signal prediction. To authors knowledge one of the most accurate simulation, performed in recent years, that takes into account the frequency attenuation of the medium, has been carried out by the ACORNE Collaboration[12]; their results(see Fig. (2)) shows a peak to peak amplitude of about $150 \mathrm{mPa}$ at $1 \mathrm{Km}$ 
from the interaction point for a neutrino energy of about $5 \cdot 10^{11} \mathrm{GeV}$. Their results show also an extremely well collimated acoustic pulse whose amplitude decreases of about one order of magnitude in some degrees off the perpendicular plane passing through the maximum, the so called acoustic "pancake". It is worth noting that the characteristic collimation of the acoustic bipolar pulse is the fundamental property of the signal that will allow to make astronomy: sampling the "pancake" profile it's possible to reconstruct the shower direction and thus the neutrino one. Moreover this property is also the key to reject the background with high efficiency since the other sources of noise, like surface noise or biological one, even if could mimic the pressure signal, have different geometrical shape.
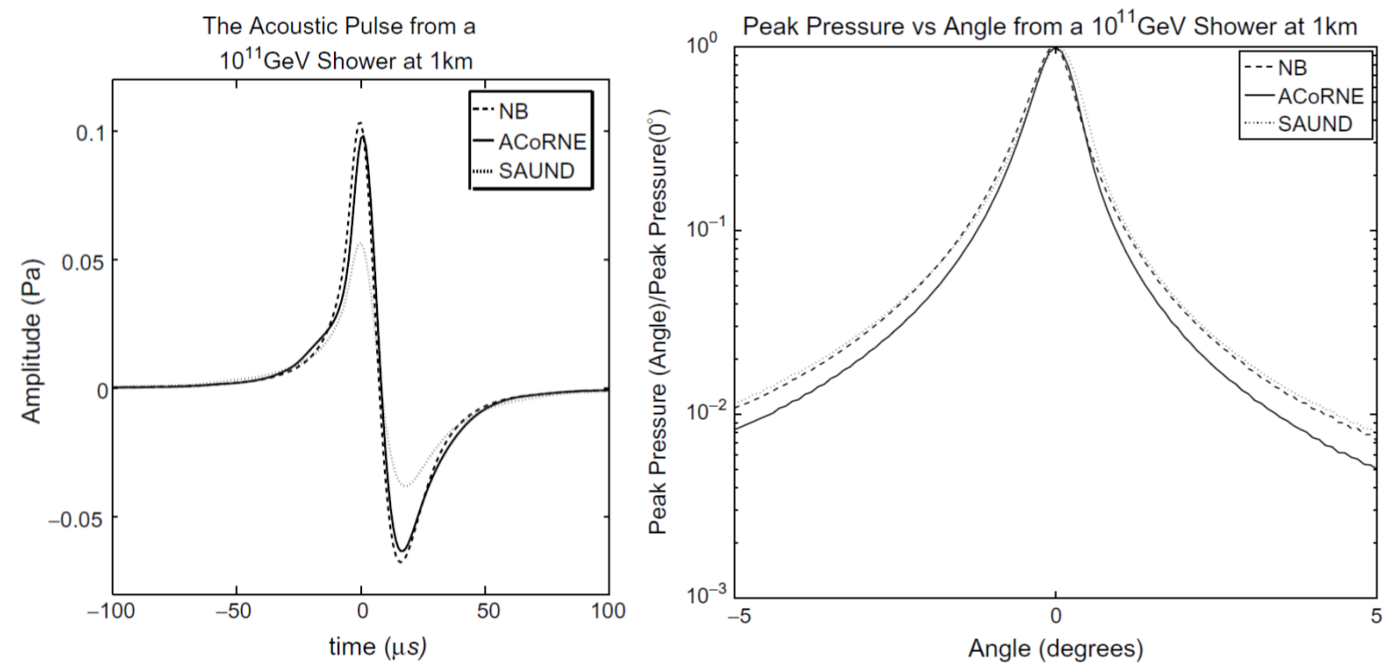

Figure 2. Acoustic pulse(left) and its angular dependency(right) for an hadronic shower of $10^{11} \mathrm{GeV}$ evaluated in a plane perpendicular to the shower axis passing to the shower maximum(taken from [12]).

\section{Acoustic infrastructures in Mediterranean sea}

In the framework of the activities of different underwater Cherenkov neutrino telescopes, many studies have been carried out on the acoustic properties of the medium, on the sensors technology development and on their calibration. The AMADEUS[13] group (ANTARES Modules for Acoustic Detection Under the Sea) has deployed a system, designed principally as acoustic positioning system of the ANTARES neutrino telescope, made of both commercial and self-made piezoelectric sensor as well as self-made hydrophones. The AMADEUS system is fully integrated into the ANTARES acquisition system. It has been exploited to monitor the deep sea noise in the site[14], to identify and trace different sources of underwater noise, of anthropic and biological origin, and to performed useful comparisons between different technological solutions for underwater acoustic sensors tested in real environmental conditions. In 2005 the NEMO[15] Collaboration deployed OvDE (Ocean Noise Detection Experiment) [16] at the NEMO test site, $2000 \mathrm{~m}$ depth, $25 \mathrm{~km}$ east of the Sicily coast, composed of four commercial hydrophones arranged on a pyramidal-shaped. All data, sampled at $96 \mathrm{kHz}$ and 24 bits, were transmitted in real-time and recorded for 5 minutes every hour. OvDE performed, for the first time, the underwater noise study in deep Mediterranean sea; data acquired allowed to characterize the deep sea noise as function of time and of weather conditions as well as in presence of ships 
and biological sources (see Fig. (3)). The study of acoustic signals of biological origin had important drawbacks in bioacoustic. The NEMO collaboration deployed in 2013 a prototype of a Cherenkov detection unit (the NEMO phase II tower [17]) that included an acoustic positioning system. This positioning system was developed and realized by the SMO Collaboration[18]: it was composed by 18 acoustic sensors continuously sampled at $192 \mathrm{kHz} / 24$ bit and transmitted to shore for real time analysis. In such NEMO-SMO detector three different acoustic sensors were employed: 14 SMID TR-401 hydrophones, two free flooded ring hydrophones, model Sensor Technology Ltd SX-30, and two custom piezo-sensors. The SMO SMID hydrophones sensitivity (see Fig. (3)-right), as function of the pressure, changes of about $1 \mathrm{~dB}$ and are the first devices calibrated at target pressure using a novel procedure developed in collaboration with the NATO Undersea Research Center of La Spezia (Italy). These sensors were also time calibrated taking into account both acquisition electronic and ceramic latency [19].
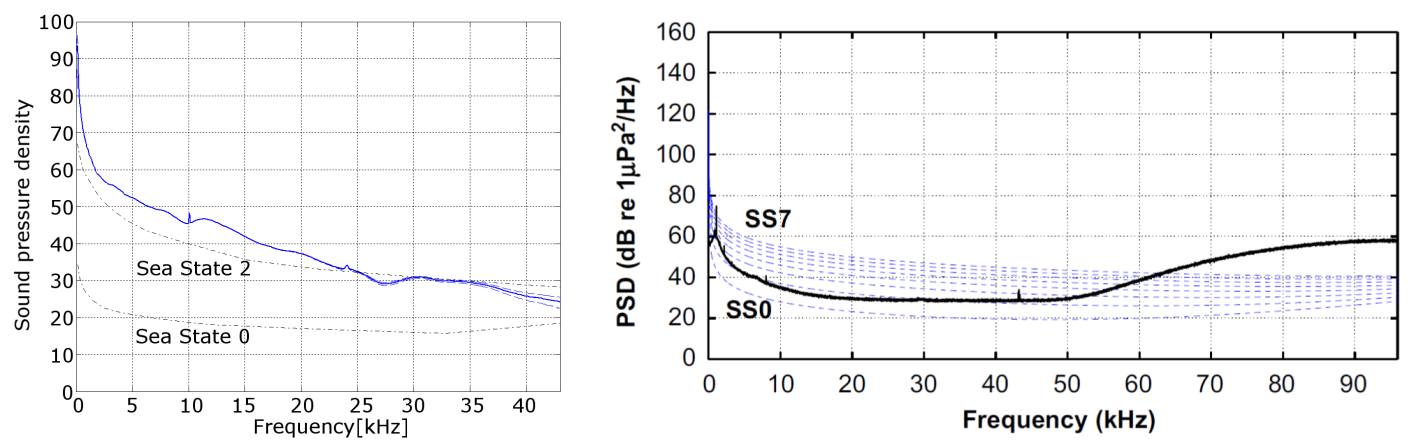

Figure 3. Noise power spectral density $\left(\mathrm{dB}\right.$ re $\mu \mathrm{Pa}^{2} / \mathrm{Hz}$ ) measure by $\mathrm{O} v$ de at $2000 \mathrm{~m}$ depth (Left). Power spectral density of the intrinsic noise of the NEMO-SMO SMID hydrophone (right).

ANTARES, NEMO, NESTOR [20] and other European Collaborations merged their effort to build an European large scale Cherenkov Neutrino Telescope forming the KM3NeT Collaboration [21]. The work performed in the recent years has been focused in the development of the digital optical module (DOM [22]) and the Detection Unit (DU) layout optimization for different fields of neutrino physics (see Fig. (4)). The aim of the KM3NeT Collaboration is to deploy two different setup, based on the same technology, in the Mediterranean sea:

- ORCA[23] a dense Megaton detector with 20m horizontal spacing between adjacent DU and a vertical inter DOM space of about $6 \mathrm{~m}$, mainly focused on the low energy $(\mathrm{E}>10 \mathrm{GeV})$ neutrino detection and neutrino mass hierarchy studies.

- ARCA[23] a neutrino detector with 100m horizontal spacing between adjacent DU and a vertical inter DOM space of about 36m, optimized for high energy neutrino detection and astroparticle studies.

Both setup will share the same basic technology and will use the DOM as building block of their DU; this means that their acoustic positioning system[24] could be use as pilot experiment for acoustic neutrino detection.

\section{Future perspective}

In view of the future availability of huge hydrophones array, like the one expected to be used as acoustic positioning system of neutrino Cherenkov detectors, simulation studies have been carried 


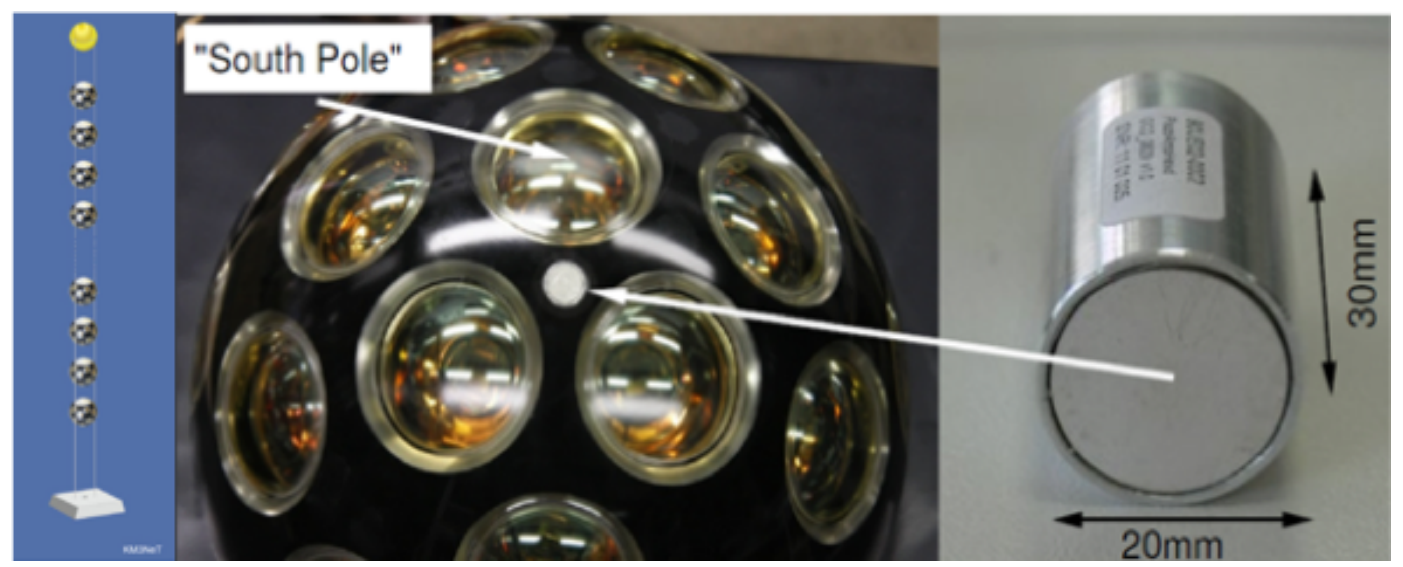

Figure 4. Scheme of the KM3NeT detection unit(left). Picture of the digital optical module(right) with detail of the internal piezoelectric sensor.

out by the AMADEUS [25] and ACORNE [26] groups, to evaluate the sensitivity of the acoustic technique as a function of sensors density and detection thresholds. The AMADEUS group simulated a randomly sparse sensor array with a threshold of $5 \mathrm{mPa}$ and evaluated the effective volume as a function of the sensor density (see Fig. (5)); they found an optimal density of about 200 sensors per $\mathrm{km}^{3}$. The ACORNE group has taken into account the realistic water properties, principally refraction and evaluated two different configurations (see Fig. (5)): a small dense array of $1 \mathrm{~km}^{3}$ containing 1000 hydrophones with a threshold of $35 \mathrm{mPa}$ and a huge $1500 \mathrm{~km}^{3}$ sparse array of 100 sensors per $\mathrm{km}^{3}$ with a threshold of $5 \mathrm{mPa}$. ACORNE results show that GZK neutrinos could be detected only with this second configuration.
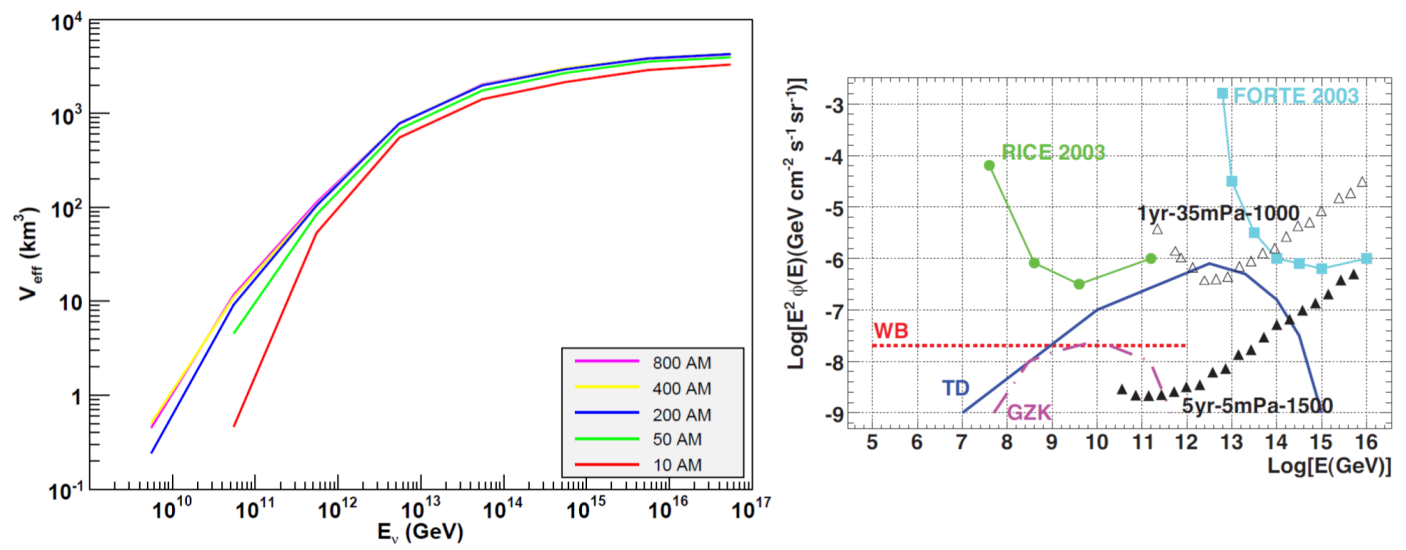

Figure 5. Left: effective volume as a function of the neutrino energy for different sensor density; the simulated instrumented volume is $1 \mathrm{~km}^{3}$. Right: the predicted limits on neutrino flux for different detector configurations, for details refer to [26] 


\section{Conclusions}

In last years many activities focused on signal simulation, sensor development and calibration have been carried out. Fast code for acoustic neutrino signal generation and propagation are at present available. Commercial hydrophones sensitivity, in the frequency range of interest for the acoustic neutrino detection, reaches the limit of underwater noise background in relatively quite sites and procedures to calibrate sensors as a function of pressure, have been developed. In next years KM3NeT Collaboration will deploy a $\mathrm{km}^{3}$ scale optical Cherenkov detector in the Mediterranean sea. Its acoustic positioning system will be the biggest underwater acoustic array suited to detect UHE neutrino interactions and will allow to validate and improve the background rejection algorithms as well as the reconstruction techniques. The acoustic detection of neutrinos in the energy range $10^{18} \mathrm{eV}-10^{21} \mathrm{eV}$ will require further improvements in signal detection: it will be needed to lower the energy threshold for neutrino detection, to define a specific detector layout and to instrument a much bigger active volume.

\section{References}

[1] The Baikal homepage (official), http://baikalweb.jinr.ru/

[2] The IceCube homepage (official), http://icecube.wisc.edu/

[3] The ANTARES homepage (official), http://antares.in2p3.fr/

[4] G.A. Askaryan et al, Nuclear Instruments and Methods, 164, 267 (1979)

[5] G.T. Zatsepin and V.A. Kuz'min, JETP Letters 4, 78 (1966)

[6] L.A. Anchordoqui et al., Physics Review Letter D 76, 123008 (2007)

[7] M. Ahlers et al., Astroparticle Physics 34, 106 (2010)

[8] R. Gandhi et al., Physics Review Letter D 58, 093009 (1998)

[9] R. Gandhi et al., Astroparticle Physics 5, 81 (1996)

[10] A. B. Migdal, Phys. Rev. 103, 1811 (1956)

[11] V. Niess et al., Astroparticle Physics 26, 243 (2006)

[12] S.Bevan et al., Astroparticle Physics 28, 366 (2007)

[13] J. A. Aguilar et al., Instrumentation and Methods A 626-627, 128 (2011)

[14] F. Simeone et al., Proceedings of the 31st ICRC conference arXiv:0908.0862v2 (2009)

[15] G. Riccobene et al., Nuclear Instruments and Methods A, 604604 (2009) 149

[16] G. Riccobene et al., e-Print, arXiv:0804.2913, (2008)

[17] T. Chiarusi et al., Journal of Instrumentation 9, C03045 (2014)

[18] F. Simeone et al., Proceedings of the MASS Conference DOI:10.1109/MASS.2011.107, (2011)

[19] S. Viola et al., Nuclear Instruments and Methods in Physics Research A 725, 207 (2013)

[20] J. Carr et al., New Journal of Physics 6 Issue 1, 112 (2004)

[21] The KM3NeT homepage (official), http://www.km3net.org/

[22] S. Adrián-Martínez et al., Eur. Phys. J. C 74, 3056 (2014)

[23] S Adrián-Martínez et al, Journal of Physics G, 43 no.8, 084001 (2016)

[24] S. Viola et al., Proceedings of Science ICRC2015 1169 (2016)

[25] Doctoral thesis, T. Karg, arXiv:astro-ph/0608312, (2006)

[26] Doctoral thesis, J. Perkin, arXiv:0801.0991, (2008) 